\title{
Sonohisterografía \\ Un recurso diagnóstico en pacientes con hemorragia uterina anormal
}

\author{
Eyder Burbano Adrada, MD*
}

\section{RESUMEN}

INTRODUCCION: Instilar líquido en la cavidad endometrial, al momento de realizar la ecografía transvaginal para determinar como mejora la observación de su estructura, fue el objetivo del presente estudio.

MATERIAL Y METODOS: Se incluyeron 100 pacientes entre 36 y 53 años de edad, con hemorragia uterina anormal, sin respuesta a los tratamientos hormonales convencionales, anormalidades en la ecografía transvaginal como pérdida de la interfase endometriomiometrio o distorsión del contorno endometrial

RESULTADOS: El procedimiento mejoró la precisión diagnóstica, se observó con claridad 14 pólipos endometriales, 9 miomas submucosos, 19 miomas que hacían contacto con la cavidad endometrial sin deformarla, 23 miomas intramurales que distorsionaban la línea endometrial, 8 pacientes con engrosamiento irregular del endometrio, 22 pacientes con engrosamiento miometrial por focos de adenomiosis o miomatosis difusa, en 5 pacientes no se lograron imágenes satisfactorias por alteración de la elasticidad miometrial.

CONCLUSIONES: La sonohisterografía mejora la visualización del útero y del endometrio, el liquido actúa como una ventana acústica que permite observar con mayor claridad las anormalidades presentes. Este procedimiento es seguro, fácil de realizar, no doloso y especialmente útil para el diagnóstico de miomas submucosos y pólipos endometriales.

PALABRAS CLAVES: Sonohisterografía, cavidad endometrial, miometrio, endometrio, pólipo, mioma.

\section{SUMMARY}

INTRODUCTION: To install liquid in the endometrial cavity in the moment of accomplishing the transvaginal ecography to determine how improves the observation of its structure, it was the objective of the present study.

MATERIAL AND METHODOS: There were included 100 patients betwieen 36 and 53 years old, with abnomal uterine bleeding, without response to the conventional hormonal treatments, abnormalities in the transvaginal ecography as last of the endometrio-miometrio interface or distortion of the endometrial contour.

RESULTS: The procedure improved the diagnostic precision, it was observed with clarity 14 endometrial polips, 9 submocous miomas, 19 miomas that were making contact with the endometrial cavity without distorting it, 23 intramural miomas that distorted the endometrial line, 8 patients with irregular enlargement by focuses of diffuse adenomiosis, in 5 patients were not achieved satisfactory images by alteration of the miometrial elasticity.

CONCLUSIONS: The sonohysterography improves the visualization of the uterus and of the endometrio, the liquid acts as an acoustic window that permits to gbsrve with greater clarity the present abnormalities. This procedure is sure, easy of accomplishing, not painful and especially useful for the diagnosis of submucous miomas and endometrial polys.

KEY WORDS: Sonohysterography, endometrial cavity, miometrio, endometrio, polyps, mioma.

\section{Introducción}

La ecografía transvaginal con su alta resolución y la mayor aproximación al útero y sus anexos, ha demostrado ser un método diagnostico de gran utilidad en la observación de patología a nivel de miometrio y cavidad endometrial; sin embargo en ocasiones no resulta fácil distinguir entre un pólipo o mioma submucoso de

* Ginecobstetra del Centro de Diagnóstico Perinatal. Clínica del I.S.S. Clínica Comsalud. Popayán Colombia un engrosamiento o hiperplasia endometrial que expande la cavidad endometrial o distinguir entre una tumoración que distorsiona o desplaza la línea endometrial estando confinada al endometrio o proyectándose desde el miometrio, debido a que la lesión y el tejido que la rodea pueden tener características sonoacústicas similares (1)

Estas dificultades o limitaciones de la ecografía transvaginal pueden ser superadas con la sonohisterografía contrastada. Al instilar líquido en la cavidad endome- 
trial mediante un catéter de inseminación intrauterina (2), el líquido distiende la cavidad y delimita su estructura, actuando como una ventana acústica que permite observar con mayor precisión las alteraciones que se puedan presentar en la cavidad endometrial o en el miometrio.

\section{Materiales y métodos}

El presente estudio fue realizado entre Noviembre de 1993 y Mayo de 1998 para lo cual se dispuso de un equipo de ultrasonido marca Aloka D 500 , con transductor vaginal de $5 \mathrm{MHz}$ y una cámara impresora Aloka SSZ300S y a partir de Noviembre de 1995 un equipo Aloka Flexus SSD-1100 con transductor vaginal, mecánico de 5 y $7.5 \mathrm{MHz}$ con cámara impresora Sony UP-890MD.

Para instilar el líquido en la cavidad uterina se utilizó catéteres de inseminación intrauterina de $2.3 \mathrm{~m} . \mathrm{m}$. KDF2.3 (Unimar).

Las 100 pacientes en edades comprendidas entre 36 y 53 años (tabla No. 1), con hemorragia uterina anormal, todas con legrado biopsico previo (tabla No. 2), sin respuesta a tratamientos hormonales convencionales, hallazgos a la ecografía transvaginal tales como pérdida de la interfase endometrio-miomatrio, alteración del contorno endometrial, engrosamiento, distorsión o desplazamiento de la línea endometrial, 37 pacientes con ecografía pélvica transabdominal y 6 con ecografía transvaginal informadas como normales.

Antes del examen, la paciente vacía la vejiga, el examen es realizado con la paciente en posición de litotomía. El procedimiento se inicia localizando el cuello uterino mediante un especulo, se realiza asepsia con solución de yodopolividona, se fija el cuello con una pinza de tentáculo (pinza de Schroeder), se introduce el catéter a través del canal cervical hasta el tercio medio de la cavidad endometrial, luego con un esparadrapo se fija el catéter a la pinza, de esta manera se puede retirar el espéculo sin que el catéter se salga de la cavidad uterina, el transductor vaginal se introduce hasta el fondo de saco posterior, luego se conecta el catéter a una jeringa de 20c.c. con solución salina estéril, la mano derecha del investigador (E.B) maneja el transductor y la izquierda congela las imágenes que se van a imprimir, en tanto que la enfermera (A.L.C) va inyectando lentamente la solución salina a la cavidad endometrial. El útero es escaneado en sus planos axiales y longitudinales. Las imágenes son comparadas con la ecografía transvaginal previa, el procedimiento es además grabado en equipo de video VHS Panasonic.

\section{Resultados}

Durante los 66 meses del estudio se incluyeron 100 pacientes con las características establecidas, con los siguientes hallazgos (tabla No. 3 ): 14 pólipos endometriales, 9 miomas pediculados que hacían protrución en la sonolúcida cavidad distendida con la solución salina, 19 miomas que hacían contacto con la cavidad endometrial sin distorsionarla, 23 miomas submucosos que distorsionaban la cavidad endometrial, 8 pacientes con engro- samiento irregular del endometrio, 22 pacientes con engrosamiento difuso de una de las paredes del útero por focos de adenomiosis o miomatosis difusa. En solo 5 pacientes no se lograron imágenes satisfactorias por la falta de elasticidad del miometrio o por adherencias intrauterinas.

Tabla No. 1

DISTRIBUCION DE LAS PACIENTES EN GRUPOS ETÁREOS

\begin{tabular}{|cc|}
\hline Edad & No. de pacientes \\
\hline $36-40$ & 14 \\
$41-45$ & 21 \\
$46-50$ & 36 \\
$51-53$ & 29 \\
\hline
\end{tabular}

Tabla No. 2

INFORMES DE ANATOMIA PATOLOGICA DEL LEGRADO BIOPSICO PREVIO

Atrofia

31

Fase secretora

Estímulo hormonal 27

Muestra insuficiente 13

Hiperplasia simple

Hiperplasia compleja

Hiperplasia glandular típica con metaplasia escamosa acantósica

Hiperplasia focal

Tabla No. 3

HALLAZGOS EN LA SONOHISTEROGRAFIA

Pólipos endometriales

14

Miomas submucosos pediculados

Miomas en contacto endometriall

9

Miomas que deforman endometrio

23

Engrosamiento irregular del endometrio

8

Adenomiosis/Miomatosis difusa

22

Utero rígido 
Figuras 1 y 2

UTERO DE TAMAÑO NORMAL, CON IMAGEN CENTRAL DE ECOGENICIDAD SEMEJANTE A LA DE LAS PAREDES DEL UTERO, LA SONOHISTEROGRAFIA PERMITIO OBSERVAR UN PEQUEÑO MIOMA DE 1.8 CM. QUE HACIA CONTACTO CON LA CAVIDAD ENDOMETRIAL DEFORMANDOLA.
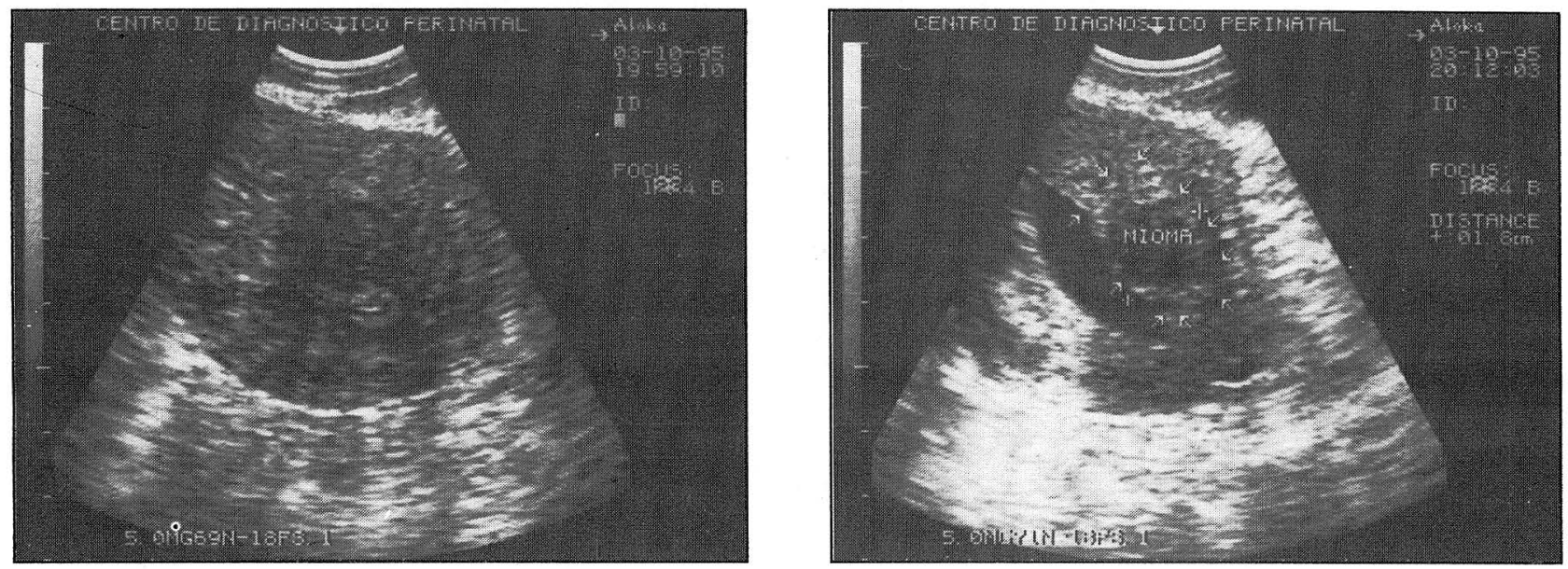

Figuras 3 y 4

LA SONOHISTEROGRAFIA PERMITIO LA CLASIFICACION DE ESTE MIOMA DE 18X24 MM. COMO UN MIOMA PEDICULADO, IDEAL PARA MIOMECTOMIA POR HISTEROSCOPIA.
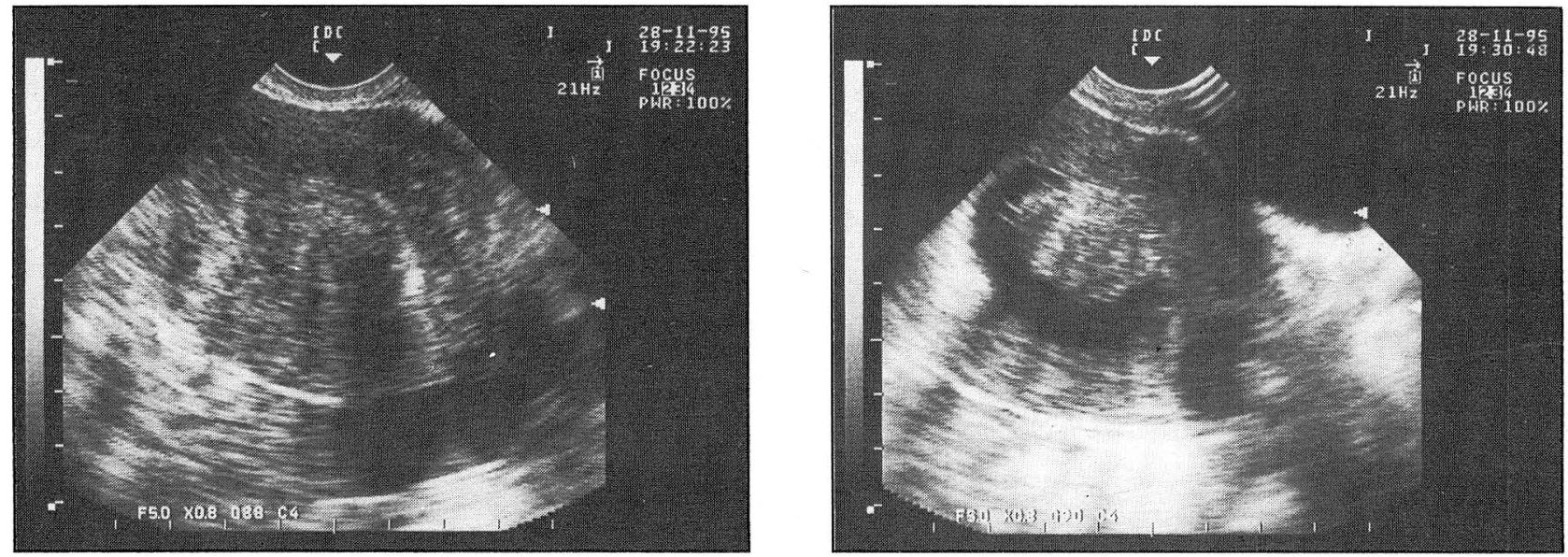

Figuras 5 y 6

POLIPOS ENDOMETRIALES, QUE EN LA MAYORIA DE LAS VECES SE DIAGNOSTICAN POR ECOGRAFIA COMO HIPERPLASIAS ENDOMETRIALES
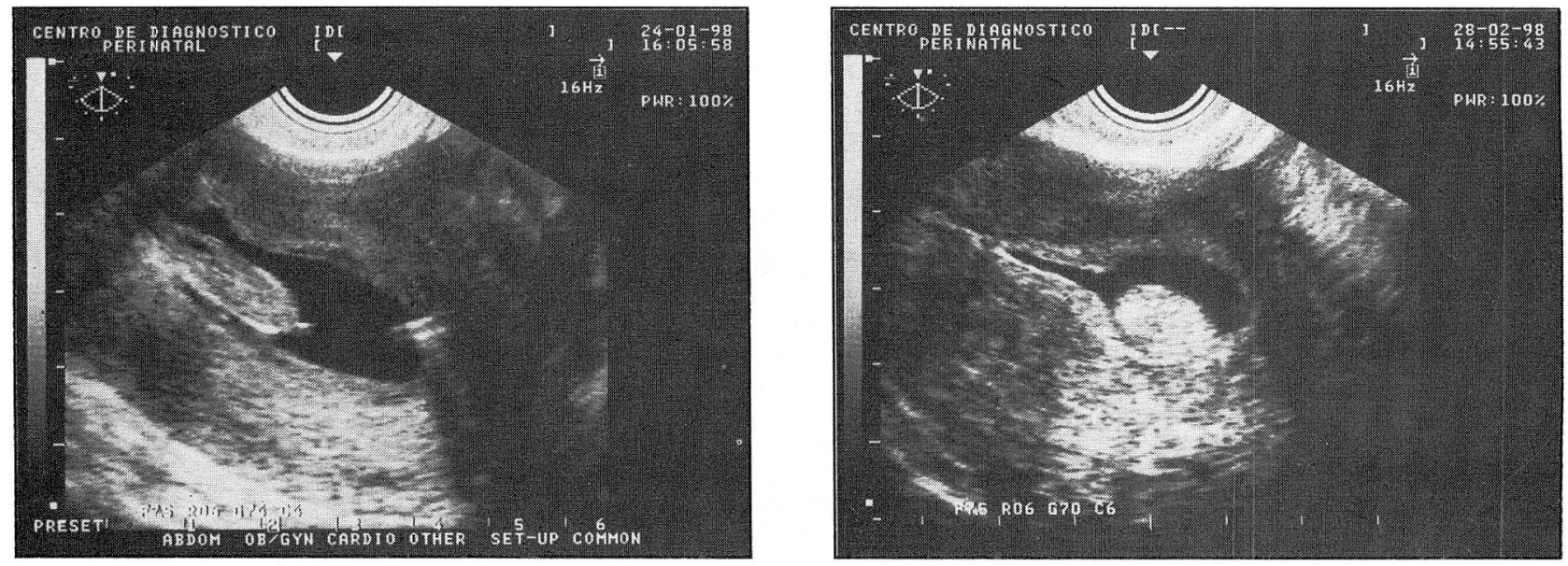
Figuras 7 y 8

OTROS TIPOS DE MIOMAS QUE DISTORSIONAN LA CAVIDAD ENDOMETRIAL.

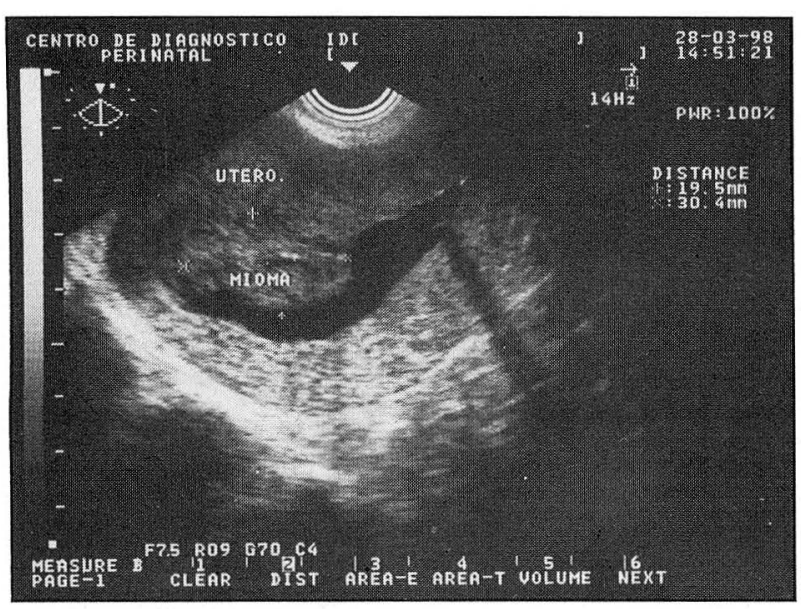

\section{Conclusiones}

Con la histerosonografía contrastada se logra mejorar la visualización tanto de la cavidad endometrial como del miometrio, al entrar el líquido a la cavidad endometrial la distiende y delimita sus estructuras, de esta manera la solución salina dentro del útero se convierte en una ventana acústica que permite observar con mayor nitidez las anormalidades que se puedan presentar (3). Este

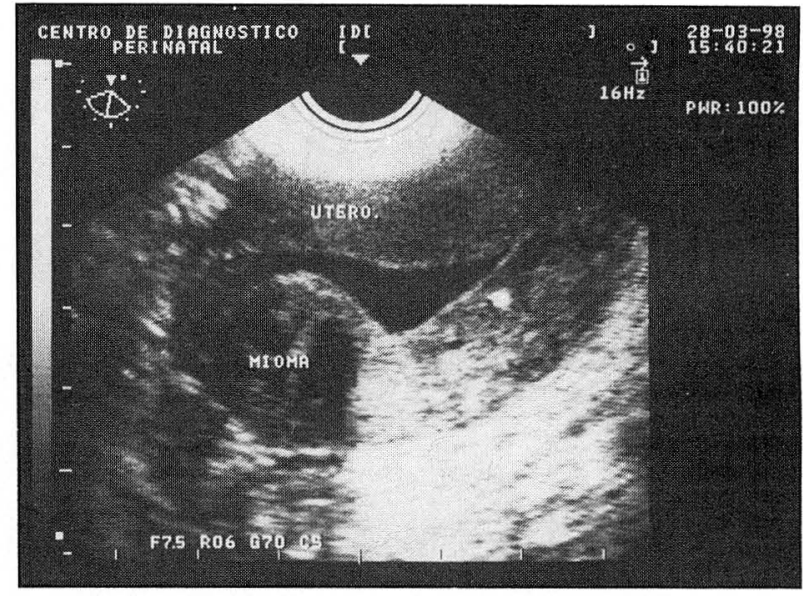

procedimiento demostró ser seguro, fácil de realizar, no doloroso y especialmente útil para detectar pólipos endometriales, miomas submucosos (4), además permite valorar la capacidad de distensión del miometrio y es un método que puede ser de gran utilidad en la valoración prequirúrgica de pacientes a quienes se les va a realizar procedimientos como histeroscopia, ablación endometrial, miomectomías, o polipectomías.

\section{BIBLIOGRAFIA}

1. Turner TT, Berman AM, Topel HC.Improved demonstration of endometril polyps and submucous myomas using saline-enhanced vaginal sonohysterography. The Jounal of the American Association of Gynecologic Laparoscopists 1995; 2:421-25

2. Goldstein SR.Use of ultrasohysterography for triage of perimenopausal patients with unexplained uterine bleeding 1994 . 170:565-70.
3. Syrop C, Sahakian V. Transvaginal sonographic detectiion of endometria polyps with fluid contrast aumentation. Obstet Gynecol 1992;79:041-3

4. Timor-Tritsch IE. Postgraduate Course: Vaginosography in Gynecology: Applications World Congress of Gynecologic Endoscopy. AAGL $22^{\circ}$ Annual Meeting, San Francisco, California, November 1993. 